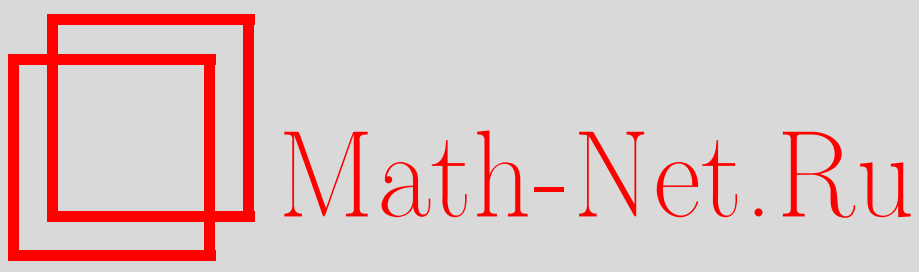

А. М. Седлецкий, Необходимое условие равномерной минимальности системы экспонент в пространствах $L^{p}$ на прямой, Матем. сб., 2001, том 192, номер 11, 137-156

DOI: https://doi.org/10.4213/sm613

Использование Общероссийского математического портала Math-Net.Ru подразумевает, что вы прочитали и согласны с пользовательским соглашением

http: //www. mathnet.ru/rus/agreement

Параметры загрузки:

IP: 52.205 .19 .152

26 апреля 2023 г., 10:28:00 


\section{А.М. Седлецкий}

\section{Необходимое условие равномерной минимальности системы экспонент в пространствах $L^{p}$ на прямой}

\footnotetext{
В статье получено необходимое условие равномерной минимальности системы весовых экспонент

$$
\exp \left(-i \lambda_{n} t-a|t|^{\alpha}\right), \quad a>0, \quad \alpha>1,
$$

в пространствах $L^{p}, 1 \leqslant p<\infty$, и $C_{0}$ на прямой и на полупрямой. Условие формулируется в терминах индикатора целой функции порядка $\beta=\alpha /(\alpha-1)$, нули которой совпадают с последовательностью $\lambda_{n}$. С помощью него доказано, что среди известных полных и минимальных систем такого вида в указанных пространствах базисов нет.

Библиографиия: 20 названий.
}

\section{§1. Введение}

Под $B\left(B_{+}\right)$будем понимать любое из пространств $L^{p}=L^{p}(\mathbb{R}), C_{0}=C_{0}(\mathbb{R})$ $\left(L_{+}^{p}=L^{p}\left(\mathbb{R}_{+}\right), C_{0}^{+}=C_{0}\left(\mathbb{R}_{+}\right)\right), 1 \leqslant p<\infty$, где $C_{0}(\mathbb{R})\left(C_{0}\left(\mathbb{R}_{+}\right)\right)$обозначает снабженное sup-нормой пространство непрерьвных на $\mathbb{R}\left(\right.$ на $\left.\mathbb{R}_{+}=[0,+\infty)\right)$ функций таких, что $f(t) \rightarrow 0, t \rightarrow \pm \infty(t \rightarrow+\infty)$. В статье получено необходимое условие равномерной минимальности в $B$ и $B_{+}$системы весовых экспонент

$$
\left(e_{n}(t)\right)_{n=0}^{\infty}:=\left(e^{-i \lambda_{n} t} \exp \left(-a|t|^{\alpha}\right)\right)_{n=0}^{\infty}, \quad a>0, \quad \alpha>1,
$$

где $\lambda_{n} \in \mathbb{C},\left|\lambda_{n+1}\right| \geqslant\left|\lambda_{n}\right|, \Lambda=\left(\lambda_{n}\right)_{n=0}^{\infty}$.

Интерес к аппроксимационньм свойствам весовых систем экспонент

$$
\left(e^{-i \lambda_{n} t} g(t)\right)_{n=0}^{\infty}, \quad g \in L^{2}, \Lambda=\left(\lambda_{n}\right)_{n=0}^{\infty},
$$

в пространстве $L^{2}$ связан с теоремой Винера [1], утверждаюшей, что линейные комбинации сдвигов $f(t+\lambda), \lambda \in \mathbb{R}$, функции $f \in L^{2}$ плотны в $L^{2}$ тогда и только тогда, когда преобразование Фурье $\widehat{f} \neq 0$ почти всюду. Эквивалентная формулировка $($ с $g=\widehat{f})$ такова: линейные комбинации функций

$$
\left(e^{-i \lambda t} g(t)\right)_{\lambda \in \mathbb{R}}, \quad g \in L^{2},
$$

плотны в $L^{2}$ тогда и только тогда, когда $g \neq 0$ почти всюду.

При фиксированной функции $g$ семейство (3) представляет собой континуальное множество функций, тогда как обычно в теории аппроксимации имеют дело

(C) А.М. СЕдлецкий 2001 
с системами (т.е. с последовательностями) функций. Поэтому переход от семейства (3) к системе (2), позволяющий более экономно использовать множество параметров $\lambda$, согласуется с традициями классической теории аппроксимации. При этом естественно искать последовательности $\Lambda$, не только являющиеся неплотными в $\mathbb{R}$, но и такие, что $\lambda_{n} \rightarrow \infty$. Следующая теорема показывает, что такой выбор возможен далеко не всегда.

TeOpema A [2]. Пусmb $g \in L^{2} u$

$$
|g(t)| \geqslant \exp (-\omega(|t|)), \quad t \in \mathbb{R},
$$

әде $\omega(t)$ - положсительная неубывающая функция на $\mathbb{R}_{+}$с условием

$$
\int_{\mathbb{R}_{+}} \frac{\omega(t)}{1+t^{2}} d t<+\infty .
$$

Тогда если последовательность $\Lambda \subset \mathbb{R}$ неплотна в $\mathbb{R}$, то система (2) неполна в $L^{2}$.

Таким образом, для изучения полных в $L^{2}$ систем $(2)$ с неплотными в $\mathbb{R}$ последовательностями $\Lambda$ следует ограничиться весами $g(t)$, убывающими достаточно быстро. В настоящее время наиболее исследованным является случай веса

$$
g(t)=\exp \left(-a|t|^{\alpha}\right), \quad a>0, \quad \alpha>1,
$$

соответствуюшего системе (1). В статьях [3]-[6] получен ряд условий (как достаточных, так и необходимых) полноты системы (1) в $L^{2}$. Однако все полные системы (1), встречающиеся в этих статьях, не являются минимальными. Впервые полная и одновременно минимальная в $L^{2}$ система (1) с $\alpha=2$ была предъявлена в [7]. Этот результат был расширен в [8] и [9], где исследован уже некоторый класс полных и минимальных систем (1) в $L^{2}$, но снова при $\alpha=2$. В [10] для произвольных $a>0, \alpha>1$ построен класс целых функций, зависящих от комплексного параметра $\mu$, и описаны значения $\mu$, обладающие свойством: если $\Lambda$ есть последовательность корней функции этого класса, то соответствующая система (1) полна и минимальна в $L^{p}\left(L_{+}^{p}\right), 2 \leqslant p<\infty$. В [11] для более широкого класса целых функций этот результат распространен на пространства $L^{p}, C_{0}\left(L_{+}^{p}, C_{0}^{+}\right), 1 \leqslant p<2$. В итоге, к настоящему моменту для всех $a>0, \alpha>1$ мы располагаем полными и минимальньми (одновременно) системами (1) во всех пространствах $B\left(B_{+}\right)$.

Стоит отметить, что в работах [3]-[6] речь, в основном, идет о вещественных последовательностях $\Lambda$ (хотя некоторые результаты верны и для комплексных). Скорее всего, это вызвано некоторой оглядкой на первоисточник [1]. Но все последовательности $\Lambda$, фигурирующие в статьях [7]-[11], уже являются комплексными, и этому есть свое объяснение. Дело в том, что если, например, $\beta=\alpha /(\alpha-1)=2 k$, $k \in \mathbb{N}$, а $\Lambda \subset \mathbb{R}$, то система (1) не может быть одновременно полной и минимальной в $L^{2}$ (подробно об этом сказано в [10]).

Возникает естественньй вопрос: сушествуют ли базисы в пространствах $B$ $\left(B_{+}\right)$среди имеющихся полных и минимальных систем $(1)$ в этих пространствах? Мы ответим на этот вопрос, найдя необходимое условие сначала полноты и минимальности, а затем и равномерной минимальности системы $(1)$ в $B\left(B_{+}\right)$. 


\section{§2. Системы элементов в банаховом пространстве}

Следующие определения и факты имеются, например, в [12].

Система $\left(e_{n}\right)$ элементов банахова пространства $B$ называется:

а) полной, если $\overline{\operatorname{span}}\left(e_{n}\right)=B$;

б) минимальной, если для любого $n$

$$
\rho_{n}:=\operatorname{dist}\left(e_{n}, \overline{\operatorname{span}}\left(e_{k}\right)_{k \neq n}\right)>0
$$

в) равномерно минимальной, если $\rho_{n} \geqslant \delta\left\|e_{n}\right\|$ для всех $n$, где $\delta>0$ не зависит от $n$;

г) базисом, если для любого элемента $x \in B$ существует единственный ряд по системе $\left(e_{n}\right)$, сходящийся к $x$ :

$$
x=\sum_{n} c_{n} e_{n}, \quad c_{n}-\text { скаляры. }
$$

Неполнота системы $\left(e_{n}\right)$ равносильна существованию нетривиального функционала $h \in B^{*}$, аннулирующего систему $\left(e_{n}\right):\left(h, e_{n}\right)=0$.

Минимальность системы $\left(e_{n}\right)$ равносильна сушествованию системы сопряженных функционалов $\left(h_{n}\right) \in B^{*}$, т.е. таких, что $\left(h_{m}, e_{n}\right)=\delta_{m n}$. Систему $\left(h_{n}\right)$ называют также биортогональной системой к системе $\left(e_{n}\right)$.

Если минимальная система полна, то биортогональная система единственна. В этом случае равномерная минимальность системы $\left(e_{n}\right)$ равносильна условию

$$
\sup _{n}\left\|e_{n}\right\| \cdot\left\|h_{n}\right\|<+\infty
$$

Если $\left(e_{n}\right)$ - базис пространства $B$, то система $\left(e_{n}\right)$ полна и равномерно минимальна.

\section{§ 3. Необходимое условие полноты и минимальности}

Всюду в дальнейшем $a>0, \alpha>1,1 / \alpha+1 / \beta=1,1 \leqslant p<\infty, 1 / p+1 / q=1$, $z=x+i y=r e^{i \theta}, K(\beta, a)=\beta^{-1} \cdot(\alpha a)^{-\beta / \alpha} ; H(\theta)=K(\beta, a)|\sin \theta|^{\beta}, \theta \in \mathbb{R}$, $H_{+}(\theta)=H(\theta)$, если $\sin \theta \geqslant 0$, и $H_{+}(\theta)=0$, если $\sin \theta<0$. Через $V$ и $V_{+}$обозначаем пространства функций ограниченной вариации на $\mathbb{R}$ и $\mathbb{R}_{+}$соответственно. При фиксированных $a>0, \alpha>1$ класс целых функций

$$
F(z)=\int_{\mathbb{R}\left(\mathbb{R}_{+}\right)} e^{-i z t} \exp \left(-a|t|^{\alpha}\right) d \sigma(t),
$$

где $\sigma \in V\left(V_{+}\right)$, обозначаем через $\mathscr{F} V\left(\mathscr{F} V_{+}\right)$. Если $d \sigma(t)=f(t) d t, f \in L^{q}\left(L_{+}^{q}\right)$, то соответствуюшие классы $(5)$ обозначаем через $\mathscr{F} L^{q}\left(\mathscr{F} L_{+}^{q}\right)$. Помня, что $B^{*}=$ $L^{q}, V$ и $B_{+}^{*}=L_{+}^{q}, V_{+}$при $B=L^{p}, C_{0}$ и $B_{+}=L_{+}^{p}, C_{0}^{+}$, для введенных только что классов будем использовать единообразные обозначения $\mathscr{F} B^{*}$ и $\mathscr{F} B_{+}^{*}$.

Лемма 1. Пусть $\lambda$ - корень функиии (5), әде

a) $\sigma \in V\left(V_{+}\right)$uлu

б) $d \sigma(t)=f(t) d t, f \in L^{q}\left(L_{+}^{q}\right), 1 \leqslant q \leqslant \infty$. 
Тогда

$$
\frac{F(z)}{z-\lambda}=-i \int_{\mathbb{R}_{\left(\mathbb{R}_{+}\right)}} e^{-i z t} \exp \left(-a|t|^{\alpha}\right) g(t) d t
$$

$2 \partial e$

$$
g(t)=\exp \left(a|t|^{\alpha}+i \lambda t\right) \int_{t}^{\infty} \exp \left(-a|x|^{\alpha}-i \lambda x\right) d \sigma(x)
$$

ДокАЗАтЕльство. Достаточно записать $F(z)$ в виде

$$
F(z)=-\int_{\mathbb{R}_{\left(\mathbb{R}_{+}\right)}} e^{-i(z-\lambda) t} d t \int_{t}^{\infty} e^{-i \lambda x} \exp \left(-a|x|^{\alpha}\right) d \sigma(x),
$$

затем проинтегрировать по частям и учесть, что $F(\lambda)=0$.

ЛЕмма 2 [13; гл. 2, теорема 2.6]. Пусть $b \in \mathbb{R}, s>-1$. Тогда при $x \rightarrow+\infty$

$$
\begin{aligned}
\exp \left(a x^{\alpha}+b x\right) \int_{x}^{\infty} \exp \left(-a t^{\alpha}-b t\right) t^{s} d t & \sim \frac{1}{a \alpha x^{\alpha-1-s}}, \\
\exp \left(-a x^{\alpha}-b x\right) \int_{0}^{x} \exp \left(a t^{\alpha}+b t\right) t^{s} d t & \sim \frac{1}{a \alpha x^{\alpha-1-s}} .
\end{aligned}
$$

Лемма 3. Пусть $\lambda \in \mathbb{C}$ фиксировано и $g(t)-$ функиия (7). Тогда

1) если $\sigma \in V_{+}$, то $g(t) \in L_{+}^{q}$ при всех $1 \leqslant q \leqslant \infty$,

2) если в (7) $d \sigma(t)=f(t) d t$, то оператор

$$
f(t) \rightarrow\left(1+t^{\alpha-1}\right) g(t)
$$

ограничен в $L_{+}^{q}, 1 \leqslant q \leqslant \infty$.

ДокАЗАтЕльство. Так как $|g(t)|<C\|f\|$ при $0 \leqslant t \leqslant 1$, где норма понимается соответственно в $V_{+}$и $L_{+}^{q}$, то достаточно доказать лемму, заменив $L_{+}^{q}$ на $L^{q}(1,+\infty)$, а оператор $f(t) \rightarrow\left(1+t^{\alpha-1}\right) g(t)$ на оператор $f(t) \rightarrow t^{\alpha-1} g(t)$.

1) Пусть $b=\operatorname{Im} \lambda, v(x)=\operatorname{var}(\sigma(t): 0 \leqslant t \leqslant x)$. Тогда

$$
\|g\|=\|g\|_{L^{q}(1, \infty)}=\sup _{\varphi}\left|\int_{1}^{\infty} \varphi(t) \exp \left(a t^{\alpha}+i \lambda t\right) d t \int_{t}^{\infty} \exp \left(-a x^{\alpha}-i \lambda x\right) d \sigma(x)\right|,
$$

где верхняя грань берется по $\varphi \in L^{p}(1, \infty),\|\varphi\|=1$. Значит,

$$
\begin{aligned}
\|g\| & \leqslant \sup _{\varphi} \int_{1}^{\infty} \exp \left(-a x^{\alpha}+b x\right) d v(x) \int_{1}^{x} \exp \left(a t^{\alpha}-b t\right)|\varphi(t)| d t \\
& \leqslant \int_{1}^{\infty} \exp \left(-a x^{\alpha}+b x\right) \cdot\left\|\exp \left(a t^{\alpha}-b t\right)\right\|_{L^{q}(1, x)} d v(x) .
\end{aligned}
$$

При подходящем $A>0$ функция $\exp \left(a t^{\alpha}-b t\right)$ возрастает на полупрямой $t \geqslant A$, поэтому в случае $q=\infty$ для достаточно больших $x$ присутствуюшая в (8) норма не превосходит $C \exp \left(a x^{\alpha}-b x\right)$ и, значит, правая часть в $(8)$ конечна. Если $q<\infty$, то по лемме 2 указанная норма не превосходит $C x^{(1-\alpha) / q} \exp \left(a x^{\alpha}-b x\right)$, и снова правая часть в (8) конечна. Утверждение 1) доказано. 
2) Пусть сначала $q=\infty$. Тогда при $t>1$ по лемме 2

$$
|g(t)| \leqslant\|f\|_{\infty} \cdot \exp \left(a t^{\alpha}-b t\right) \int_{t}^{\infty} \exp \left(-a x^{\alpha}+b x\right) d x \leqslant C\|f\|_{\infty} \cdot t^{1-\alpha}
$$

и, значит, при $q=\infty$ утверждение 2 ) верно. Если же $q=1$, то

$$
\begin{aligned}
\left\|g(t) t^{\alpha-1}\right\|_{L^{1}(1, \infty)} & \leqslant \int_{1}^{\infty} \exp \left(a t^{\alpha}-b t\right) t^{\alpha-1} d t \int_{t}^{\infty} \exp \left(-a x^{\alpha}+b x\right)|f(x)| d x \\
& =\int_{1}^{\infty}|f(x)| \exp \left(-a x^{\alpha}+b x\right) d x \int_{1}^{x} \exp \left(a t^{\alpha}-b t\right) t^{\alpha-1} d t \\
& \leqslant C\|f\|_{L^{1}(1, \infty)}
\end{aligned}
$$

в силу леммы 2 с $s=\alpha-1$. При $q=1$ утверждение 2) верно.

По теореме Рисса-Торина из доказанных случаев $q=1, \infty$ следует справедливость утверждения 2 ) при всех $1 \leqslant q \leqslant \infty$. Лемма 3 доказана.

ЛЕмма 4. Пусть выполнень условия леммы 1. Тогда справедливо представление (6), где

а) $g \in L^{q}\left(L_{+}^{q}\right)$ при всех $1 \leqslant q \leqslant \infty$ или

б) $\left(1+|t|^{\alpha-1}\right) g(t) \in L^{q}\left(L_{+}^{q}\right)$.

ДокАЗАТЕльство. Случай полупрямой $\mathbb{R}_{+}$в $(5)$ сразу следует из лемм 1 и 3. Пусть интегрирование в $(5)$ ведется по всей прямой $\mathbb{R}$. Тогда в силу леммы 3 остается показать, что

a) $g \in L_{-}^{q}$ или

б) $\left(1+|\bar{t}|^{\alpha-1}\right) g(t) \in L_{-}^{q}$, где $L_{-}^{q}=L^{q}\left(\mathbb{R} \backslash \mathbb{R}_{+}\right)$.

Так как $F(\lambda)=0$, то при $t<0$ равенство $(7)$ записывается в виде

$$
g(t)=\exp \left(a|t|^{\alpha}+i \lambda t\right) \int_{-|t|}^{-\infty} \exp \left(-a|x|^{\alpha}-i \lambda x\right) d \sigma(x),
$$

и, значит, заменяя $x$ на $-x$, мы представляем $g(t)$ в виде

$$
g(t)=\exp \left(a|t|^{\alpha}-i \lambda|t|\right) \int_{|t|}^{+\infty} \exp \left(-a x^{\alpha}+i \lambda x\right) d \sigma(-x)
$$

к которому уже применима лемма 3. Из нее все и следует. Лемма 4 доказана.

Так как $\mathscr{F} L^{1} \subset \mathscr{F} V$, то непосредственным следствием леммы 4 является

Лемма 5. Пусть $\lambda-$ корень функиии $F(z) \in \mathscr{F} B^{*}\left(\mathscr{F} B_{+}^{*}\right)$. Тогда

$$
\frac{F(z)}{z-\lambda} \in \mathscr{F} B^{*}\left(\mathscr{F} B_{+}^{*}\right)
$$

Для случая $B=L^{q}$ лемма 5 доказана в [14], где содержится и лемма 1. Доказательство леммы 3 построено модификацией доказательства из [14]. 
Из леммы 5 сразу следует, что в ее условиях

$$
\frac{z-\mu}{z-\lambda} F(z)=F(z)+(\lambda-\mu) \frac{F(z)}{z-\lambda} \in \mathscr{F} B^{*}\left(\mathscr{F} B_{+}^{*}\right)
$$

Впредь вместо (1) рассматриваем более обшую систему

$$
e(\Lambda):=\left((i t)^{k-1} e^{-i \lambda_{n} t} \exp \left(-a|t|^{\alpha}\right)\right)_{k=1}^{m_{n}}, \quad n \in \mathbb{Z}_{+} ; \quad \Lambda=\left(\lambda_{n}\right),
$$

подразумевая, что каждая точка $\lambda_{n}$ в $\Lambda$ считается со своей кратностью $m_{n}$. Так как неполнота системы $e(\Lambda)$ в $B\left(B_{+}\right)$равносильна сушествованию нетривиальной целой функции $F(z)$ класса $\mathscr{F} B^{*}\left(\mathscr{F} B_{+}^{*}\right)$ такой, что $F(\Lambda)=0$ (обращение в нуль понимается с учетом кратностей), то из формулы (9) следует

ЛЕмма 6. Замена в последовательности $\Lambda$ конечного числа точек $\lambda_{n}$ конечным числом других точек $\mu_{s}$ той же суммарной кратности не нарушает полнотыл системы е $(\Lambda)$ в $B\left(B_{+}\right)$.

Лемма 7. 1) Пусть $F(z) \in \mathscr{F} V\left(\mathscr{F} V_{+}\right)$u $F(\lambda)=0$. Тогда

$$
\frac{F(z)}{z-\lambda} \in \mathscr{F} L^{q}\left(\mathscr{F} L_{+}^{q}\right)
$$

при всех $1 \leqslant q \leqslant \infty$.

2) Пусть $F(z) \in \mathscr{F} L^{q}\left(\mathscr{F} L_{+}^{q}\right), 1<q \leqslant \infty$. Тогда найдется $l=l(q) \in \mathbb{N}$ такое, что если $F\left(\lambda_{i}\right)=0, i=\overline{1, l}$, то

$$
F_{l}(z):=\frac{F(z)}{\left(z-\lambda_{1}\right) \cdots\left(z-\lambda_{l}\right)} \in \mathscr{F} B^{*}\left(\mathscr{F} B_{+}^{*}\right)
$$

для всех пространств $B\left(B_{+}\right)$.

ДокАЗАтЕльство. Утверждение 1) есть перефоормулировка части а) леммы 4.

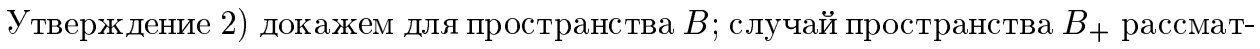
ривается аналогично.

По утверждению б) леммы 4 функция $F_{1}(z)=F(z) /\left(z-\lambda_{1}\right)$ представима в виде (6), где $\left(1+|t|^{\alpha-1}\right) g(t) \in L^{q}$.

Пусть сначала $q$ конечно. Тогда $g(t) \in L^{q / \alpha}$. Действительно, принадлежность $g(t) \in L^{q / \alpha}(-1,1)$ тривиальна, а по неравенству Гёльдера

$$
\begin{aligned}
\int_{|t|>1}|g(t)|^{q / \alpha} d t & =\int_{|t|>1}\left(|g(t)| \cdot|t|^{\alpha-1}\right)^{q / \alpha} \cdot|t|^{-q / \beta} d t \\
& \leqslant\left(\int_{|t|>1}\left(|g(t)| \cdot|t|^{\alpha-1}\right)^{q} d t\right)^{1 / \alpha} \cdot\left(\int_{|t|>1}|t|^{-q} d t\right)^{1 / \beta}
\end{aligned}
$$

Первый интеграл в правой части конечен по предположению, а второй - по условию $q>1$. Итак, $g(t) \in L^{q / \alpha}$ и $g(t) \in L^{q}$. Значит, $g(t) \in L^{s}$ при всех $s \in[q / \alpha, q]$.

Теперь, выбирая в роли $q$ число $q / \alpha$ и повторяя рассуждения, получаем, что функция $F_{2}(z)=F_{1}(z) /\left(z-\lambda_{2}\right)$ представима в виде $(6)$, где $g(t) \in L^{s}$ при всех 
$s \in\left[q / \alpha^{2}, q / \alpha\right]$. Следовательно, взяв в качестве $N$ наименьшее натуральное $n$, для которого $q / \alpha^{n} \leqslant 1$, через $N$ шагов получаем

$$
F_{N}(z)=\frac{F(z)}{\left(z-\lambda_{1}\right) \cdots\left(z-\lambda_{N}\right)} \in \mathscr{F} L^{1} \subset \mathscr{F} V
$$

где $F\left(\lambda_{i}\right)=0$. Пусть $l=N+1, F\left(\lambda_{l}\right)=0$. Тогда по утверждению 1) $F_{l}(z)=$ $F_{N}(z) /\left(z-\lambda_{l}\right) \in \mathscr{F} B^{*}$ для всех пространств $B$. Случай конечного $q$ рассмотрен.

Случай $q=\infty$ сразу сводится к рассмотренному, так как по лемме 4 функция $F(z) /\left(z-\lambda_{1}\right)$ представима в виде $(6)$ с $\left(1+|t|^{\alpha-1}\right) g(t) \in L^{\infty}$. Отсюда $g(t) \in L^{s}$ при конечном $s>1 /(\alpha-1)$. Лемма 7 доказана.

Так как каждая точка в $\Lambda$ считается со своей кратностью, то под удалением из $\Lambda$ $l$ точек будем понимать понижение на $l$ суммарной кратности некоторого конечного множества точек $\Lambda$.

Из леммы 7 сразу вытекает следующая лемма, в которой для единообразия формулировок полагаем $L^{\infty}=C_{0}, L_{+}^{\infty}=C_{0}^{+}$.

Лемма 8. Пусть система (10) неполна в $L^{s}\left(L_{+}^{s}\right)$ при некотором $1 \leqslant$ $s \leqslant \infty$. Тогда существует число $l=l(s) \in \mathbb{N}$ такое, что при удалении из $\Lambda$ $l$ точек новая система неполна во всех пространствах $L^{p}\left(L_{+}^{p}\right), 1 \leqslant p \leqslant \infty$.

Нам понадобится функиия типа Mиттаг-Леффлера (см. [15])

$$
E_{\rho}(z ; \mu)=\sum_{n=0}^{\infty} \frac{z^{n}}{\Gamma(\mu+n / \rho)}, \quad \rho>0, \quad \mu \in \mathbb{C}
$$

Лемма 9 [15]. Пусть $\rho>1, \gamma \in(\pi /(2 \rho), \pi / \rho)$. Тогда для любого $s \in \mathbb{N}$ при $r \rightarrow \infty$

$$
\begin{aligned}
& E_{\rho}(z ; \mu)=\rho z^{\rho(1-\mu)} \exp \left(z^{\rho}\right)-\sum_{k=1}^{s} \frac{z^{-k}}{\Gamma(\mu-k / \rho)}+O\left(\frac{1}{r^{s+1}}\right), \quad|\theta| \leqslant \gamma \\
& E_{\rho}(z ; \mu)=-\sum_{k=1}^{s} \frac{z^{-k}}{\Gamma(\mu-k / \rho)}+O\left(\frac{1}{r^{s+1}}\right), \quad \gamma \leqslant|\theta| \leqslant \pi
\end{aligned}
$$

Через $H_{-}^{2}$ обозначаем пространство Харди в нижней полуплоскости $y<0$. Функции $H(\theta), H_{+}(\theta)$ определены в начале $\S 3$.

Лемма 10 [16]. Пусть $G(z)-$ иелая функиия. Тогда

1) принадлежсность $G(z) \in \mathscr{F} L^{2}$ равносильна условию

$$
\|G\|^{2}:=\iint_{\mathbb{R}^{2}}|G(z)|^{2} \exp \left(-2 H(\theta) r^{\beta}\right)\left(1+|y|^{\beta / 2-1}\right) d x d y<\infty,
$$

2) принадлежсность $G(z) \in \mathscr{F} L_{+}^{2}$ равносильна тому, что $G(z) \in H_{-}^{2}$ и конечна величина $\|G\|_{+}^{2}$, равная части интеграла (11), взятой по верхней полуплоскости у $>0$. 
ТЕОРема 1. Пусть система е( $\Lambda)$ полна и минимальна в $B\left(B_{+}\right)$. Тогда существует иелая функиия $F(z)$ порядка $\beta$ такая, что:

1) индикатор $h_{F}(\theta)$ функции $F(z)$ подчиняется оценке

$$
h_{F}(\theta) \leqslant H(\theta) \quad\left(h_{F}(\theta) \leqslant H_{+}(\theta)\right), \quad \theta \in \mathbb{R},
$$

причем любой отрезок длины $\pi / \beta$ содержст хотя бъ одну точку, для которой в (12) имеет место знак равенства;

2) множсество корней функиии $F(z)$ совпадает с $\Lambda$;

3) $F(z) \notin \mathscr{F} B^{*}\left(\mathscr{F} B_{+}^{*}\right)$;

4) $F(z) /\left(z-\lambda_{0}\right) \in \mathscr{F} B^{*}\left(\mathscr{F} B_{+}^{*}\right)$.

При условии нормировки $F(\mu)=1, \mu \notin \Lambda$, функция $F(z)$ единственна.

ДокАЗАТЕЛЬСтво. Обозначим

$$
e_{n, k}=(i t)^{k-1} e^{-i \lambda_{n} t} \exp \left(-a|t|^{\alpha}\right), \quad n \in \mathbb{Z}_{+}, \quad k=\overline{1, m_{n}} .
$$

По условию система $e_{n, k}$ минимальна в $B\left(B_{+}\right)$и потому обладает биортогональной системой $h_{n, k}, n \in \mathbb{Z}_{+}, k=\overline{1, m_{n}}$, т.е. $\left(h_{m, j}, e_{n, k}\right)=1$, если $m=n, j=k$, и $\left(h_{m, j}, e_{n, k}\right)=0$ во всех остальных случаях. Отождествляя функционал с функцией, его представляющей, положим

$$
F_{0}(z)=\int_{\mathbb{R}} e^{-i z t} \exp \left(-a|t|^{\alpha}\right) h_{0, m_{0}}(t) d t, \quad h_{0, m_{0}} \in L^{q}
$$

если $B=L^{p}$; если же $B=C_{0}$, то в $(13)$ вместо $h_{0, m_{0}}(t) d t$ присутствует $d h_{0, m_{0}}(t)$, где $h_{0, m_{0}}(t) \in V$. Когда речь идет о пространстве $B_{+}$, интегрирование в (13) ведется по $\mathbb{R}_{+}$. В силу биортогональности функция $F_{0}(z)$ обращается в нуль в точках последовательности $\Lambda_{0}$, полученной из $\Lambda$ понижением на единицу кратности точки $\lambda_{0}$. Утверждается, что $\Lambda_{0}$ есть в точности множество всех корней функции $F_{0}(z)$. Действительно, если бы $F_{0}(\mu)=0, \mu \notin \Lambda$, то в силу (13) система $e\left(\Lambda_{0} \cup\{\mu\}\right)$ аннулировалась бы нетривиальньп функционалом на $B\left(B_{+}\right)$, представляемым функцией $h_{0, m_{0}}(t)$, и, следовательно, была бы неполной в $B\left(B_{+}\right)$. По лемме 6 и система $e(\Lambda)$ была бы неполной, что противоречит условию. По тем же соображениям кратность корня $\lambda_{n}$ функции $F_{0}(z)$ равна $m_{n}$ при $n \neq 0$ и равна $m_{0}-1$ при $n=0$.

Положим $F(z)=\left(z-\lambda_{0}\right) F(z)$. Тогда утверждения 2$\left.), 4\right)$ имеют место. Утверждение 3$)$ следует из полноты системы $e(\Lambda)$ в $B\left(B_{+}\right)$.

Обозначим через $\rho$ порядок $F(z)$. Из (13) следует (см., например, [16]), что $\rho \leqslant \beta$ и что индикатор функции $F(z)$ при порядке $\beta$ не превосходит $H(\theta)$. Если речь идет о пространстве $B_{+}$, то интеграл в (13) берется по $\mathbb{R}_{+}$и, значит, функция $F_{0}(z)$ ограничена в нижней полуплоскости. Значит, в этом случае $h_{F}(\theta) \leqslant 0$, $-\pi \leqslant \theta \leqslant 0$, и мы доказали (12).

Докажем единственность функции $F(z)$, нормированной условием $F(\mu)=1$, $\mu \notin \Lambda$. Пусть $G(z)$ - целая функция со свойствами, перечисленными в теореме 1, и пусть $G(\mu)=1$. Покажем, что $G(z) \equiv F(z)$.

Пусть $R(z)=G(z)-F(z) \not \equiv 0$. По предположению $R(\Lambda \cup\{\mu\})=0$ и $R(z) /\left(z-\lambda_{0}\right) \in \mathscr{F} B^{*}\left(\mathscr{F} B_{+}^{*}\right)$. В силу формулы $(9)$ функция $R(z) /(z-\mu) \in \mathscr{F} B^{*}$ 
$\left(\mathscr{F} B_{+}^{*}\right)$. Так как эта функция обрашается в нуль в точках $\Lambda$, то система $e(\Lambda)$ неполна в $B\left(B_{+}\right)$, что противоречит условию. Итак, $G(z) \equiv F(z)$.

Из доказанного утверждения 4$)$ и из леммы 7 следует, что

$$
F_{l}(z)=\frac{F_{0}(z)}{\left(z-\lambda_{1}\right) \cdots\left(z-\lambda_{l}\right)} \in \mathscr{F} L^{2}\left(\mathscr{F} L_{+}^{2}\right)
$$

при некотором $l \in \mathbb{N}$. Оставшиеся утверждения о порядке и об индикаторе достаточно доказать для функции $F_{l}(z)$. Предположим противное: $\rho<\beta$. Фиксируем отрезок $\left[\gamma_{1}, \gamma_{2}\right]=\left(\theta:\left|\theta-\theta_{0}\right| \leqslant \pi /(2 \beta)\right)$ так, чтобы $0<\gamma_{1}<\gamma_{2}<\pi$. Тогда, выбирая $\varepsilon$ из условия $0<2 \varepsilon<\min \left(H(\theta): \theta \in\left[\gamma_{1}, \gamma_{2}\right]\right)$, при подходяшем $\delta>0$ и при $r>r_{0}$ имеем

$$
\left|F_{l}(z)\right|<\exp \left(r^{\beta-\delta}\right)<\exp \left(r^{\beta}(H(\theta)-2 \varepsilon)\right), \quad\left|\theta-\theta_{0}\right| \leqslant \frac{\pi}{2 \beta} .
$$

Введем в рассмотрение функцию

$$
E(z)=\frac{1}{\beta} E_{\beta}\left(\varepsilon^{1 / \beta} z e^{-i \theta_{0}} ; 1\right),
$$

где $E_{\rho}(z ; \mu)$ - функция типа Миттаг-Леффлера. Из леммы 9 вытекает, что при $r>r_{1}$

$$
\begin{aligned}
& |E(z)|<2 \exp \left(\varepsilon r^{\beta} \cos \beta\left(\theta-\theta_{0}\right)\right) \leqslant 2 \exp \left(\varepsilon r^{\beta}\right), \quad\left|\theta-\theta_{0}\right| \leqslant \frac{\pi}{2 \beta}, \\
& |E(z)| \leqslant M<\infty, \quad \frac{\pi}{2 \beta} \leqslant\left|\theta-\theta_{0}\right| \leqslant \pi .
\end{aligned}
$$

Отсюда и из (15) вытекает, что для функции $G(z):=F_{l}(z) E(z)$ при $r>r_{2}$ верны оценки

$$
\begin{aligned}
& |G(z)|<2 \exp \left(r^{\beta}(H(\theta)-\varepsilon)\right), \quad\left|\theta-\theta_{0}\right| \leqslant \frac{\pi}{2 \beta}, \\
& |G(z)| \leqslant M\left|F_{l}(z)\right|, \quad \frac{\pi}{2 \beta} \leqslant\left|\theta-\theta_{0}\right| \leqslant \pi .
\end{aligned}
$$

Из (14) по лемме 10 следует, что $\left\|F_{l}\right\|^{2}<\infty\left(\left\|F_{l}\right\|_{+}^{2}<\infty\right.$ и $\left.F_{l}(z) \in H_{-}^{2}\right)$. Покажем, что $\|G\|^{2}<\infty\left(\|G\|_{+}^{2}<\infty\right.$ и $\left.G(z) \in H_{-}^{2}\right)$.

Введем дополняюшие друг друга до всей плоскости секторы $S_{1}=\left(z:\left|\theta-\theta_{0}\right| \leqslant\right.$ $\pi /(2 \beta))$ и $S_{2}=\left(z: \pi /(2 \beta) \leqslant\left|\theta-\theta_{0}\right| \leqslant \pi\right)$. Пусть $P_{ \pm}=(z: y \gtrless 0)$; заметим, что $S_{2} \supset P_{-}$. Тогда

$$
\|G\|^{2}=I_{1}+I_{2}=\int_{S_{1}}+\int_{S_{2}}, \quad\|G\|_{+}^{2}=I_{1}+I_{2}=\int_{S_{1}}+\int_{S_{2} \cap P_{+}}
$$

с той же подынтегральной функцией, что в (11). 
Из (17) и из того, что $\left\|F_{l}\right\|^{2},\left\|F_{l}\right\|_{+}^{2}<\infty$, следует, что в обоих случаях $I_{2}<\infty$, а в силу (16)

$$
\begin{aligned}
I_{1} & =\iint_{S_{1}}|G(z)|^{2}(1+|y|)^{\beta / 2-1} \cdot \exp \left(-2 H(\theta) r^{\beta}\right) d x d y \\
& \leqslant 4 \iint_{S_{1}}(1+|y|)^{\beta / 2-1} \cdot \exp \left(-2 \varepsilon r^{\beta}\right) d x d y<\infty .
\end{aligned}
$$

Значит, $\|G\|^{2},\|G\|_{+}^{2}<\infty$. В случае пространства $B_{+}$из свойства $F_{l}(z) \in H_{-}^{2}$ и из (17) следует, что $G(z) \in H_{-}^{+}$. По лемме $10 G(z) \in \mathscr{F} L^{2}\left(\mathscr{F} L_{+}^{2}\right)$. Значит, система $e(\mathrm{M})$, где М - последовательность корней функции $G(z)$, неполна в $L^{2}\left(L_{+}^{2}\right)$. Но М получается присоединением к $\Lambda \backslash\left(\lambda_{i}\right)_{i=0}^{l}$ бесконечного множества точек корней функции $E(z)$ (при $\rho \neq 1$ функция $E_{\rho}(z ; \mu)$ имеет бесконечное множество корней [15]). Заменяя в M $l+1$ точек точками $\lambda_{i}, i=\overline{0, l}$, имеем, что система $e\left(\mathrm{M}_{1}\right)$, где $\mathrm{M}_{1}$ получена присоединением к $\Lambda$ бесконечного множества точек, неполна в $L^{2}\left(L_{+}^{2}\right)$. В силу леммы 8 это противоречит тому, что система $e(\Lambda)$ полна в $B\left(B_{+}\right)$. Мы доказали, что $\rho=\beta$.

Осталось доказать часть утверждения 1) о знаке равенства в (12) для индикатора $h(\theta)$ функции $F_{l}(z)$. Предположим противное: при некотором $\theta_{0}$ в $(12)$ имеет место знак < для всех $\theta \in\left[\theta_{0}-\pi /(2 \beta), \theta_{0}+\pi /(2 \beta)\right]$. Сначала рассмотрим случай пространства $B$, т.е. пусть на указанном отрезке $h(\theta)<H(\theta)$. В силу непрерьвности обеих функций имеем $h(\theta)<H(\theta)-3 \varepsilon,\left|\theta-\theta_{0}\right|<\pi /(2 \beta)$, при подходящем $\varepsilon>0$. Значит, неравенство (15) верно для крайних его членов. Отсюда вытекают оценки (16), (17), а затем повторяются следующие за ними рассуждения. Они приводят к тому, что $G(z) \in \mathscr{F} L^{2}$ в случае пространства $B$.

Пусть речь идет о пространстве $B_{+}$. Тогда в правых частях $(15)$ и $(16) H(\theta)$ заменяется на $H_{+}(\theta)$, а (17) сохраняется.

Если отрезок $\left[\gamma_{1}, \gamma_{2}\right]=\left(\theta:\left|\theta-\theta_{0}\right| \leqslant \pi /(2 \beta)\right)$ лежит на $[0, \pi]$, то $H_{+}(\theta)=H(\theta)$ для $\theta \in\left[\gamma_{1}, \gamma_{2}\right]$ и сохраняются предыдущие рассуждения, основанные на $(16),(17)$ и касающиеся пространства $B$. Получаем, что $G(z) \in \mathscr{F} L_{+}^{2}$.

Если $\left[\gamma_{1}, \gamma_{2}\right] \subset[-\pi, 0]$, то в $(15)$ и (16) следует положить $H(\theta)=0$. Тогда из $(16),(17)$, а также из того, что $\left\|F_{l}\right\|_{+}^{2}<\infty$ и $F_{l}(z) \in H_{-}^{2}$, следует, что $\|G\|_{+}^{2}<\infty$ и $G(z) \in H_{-}^{2}$. По лемме $10 G(z) \in \mathscr{F} L_{+}^{2}$.

Остается разобрать случаи $0 \in\left(\gamma_{1}, \gamma_{2}\right)$ и $\pi \in\left(\gamma_{1}, \gamma_{2}\right)$. В силу аналогии ограничимся первым из них. Итак, пусть $\gamma_{1}<0<\gamma_{2}$ и $S_{+}=\left(z: 0<\arg z<\gamma_{2}\right)$. Оценка (16) сохраняется для $0<\theta<\gamma_{2}$, а при $\gamma_{1}<\theta<0$ в ней следует положить $H(\theta)=0$. Отсюда, из (17) и из того, что $F_{l}(z) \in H_{-}^{2}$, следует, что $G(z) \in H_{-}^{2}$. Пусть $I_{+}, I$ - части интеграла $(11)$, взятые по $S_{+}$и по дополнению $S_{+}$до верхней полуплоскости $y>0$. Тогда $I<\infty$ в силу свойства $\left\|F_{l}\right\|_{+}^{2}<\infty$ и (17), а для $I_{+}$ повторяется выкладка (18), показывающая, что $I_{+}<\infty$. В итоге, $\|G\|_{+}^{2}<\infty$ и по лемме $10 G(z) \in \mathscr{F} L_{+}^{2}$.

Итак, в случае пространства $B\left(B_{+}\right)$мы имеем свойство $G(z) \in \mathscr{F} L^{2}\left(\mathscr{F} L_{+}^{2}\right)$. Остается повторить концовку доказательства равенства $\rho=\beta$, что приводит к противоречию. Теорема 1 доказана.

ОПРЕДЕЛЕНИЕ. Пусть система $e(\Lambda)$ полна и минимальна в $B\left(B_{+}\right)$. Тогда целую функцию $F(z)$ со свойствами, перечисленными в теореме 1 , назовем порождающей функиией системы $e(\Lambda)$. 
ЗАмечАниЕ 1 . Пусть система $e(\Lambda)$ полна и минимальна в $L^{p}\left(L_{+}^{p}\right), 1 \leqslant p<\infty$, и пусть все точки $\lambda_{n}$, начиная с некоторой, просты. Тогда при $n>n_{1}$ формуль для биортогональной системь $h_{n}(t)=h_{n, 1}(t) \in L^{q}\left(L_{+}^{q}\right)$ имеют вид

$$
F_{n}(z):=\frac{F(z)}{F^{\prime}\left(\lambda_{n}\right)\left(z-\lambda_{n}\right)}=\int_{\mathbb{R}\left(\mathbb{R}_{+}\right)} e^{-i z t} \exp \left(-a|t|^{\alpha}\right) h_{n}(t) d t, \quad h_{n} \in L^{q}\left(L_{+}^{q}\right),
$$

где $F(z)$ - порождающая функция системы $e(\Lambda)$.

Действительно, по лемме 6 и по формуле (9) функция $F_{n}(z)$ при $n>n_{0}$ представима в виде правой части (19). Так как $F\left(\lambda_{n}\right)=1$ и $F\left(\Lambda \backslash\left\{\lambda_{n}\right\}\right)=0$, то $h_{n}(t)-$ элемент биортогональной системы к системе $e(\Lambda)$. Остается учесть, что в случае полноты и минимальности биортогональная система единственна.

ЗАмечАниЕ 2 . Если $B=C_{0}\left(B_{+}=C_{0}^{+}\right)$, то в формулах (19) следует заменить $h_{n}(t) d t$ на $d h_{n}(t)$, где $h_{n}(t) \in V\left(V_{+}\right)$.

\section{§4. Необходимое условие равномерной минимальности}

Лемма 11. Пусть функиия $F(z)$ аналитична в угле $\varphi \leqslant \arg z \leqslant \psi$ и имеет в нем порядок $\rho \in(0, \infty)$ и конечный тип при порядке $\rho$, пусть $h_{F}(\theta)$ индикатор функиии $F(z)$ при порядке $\rho$. Тогда

$$
h_{F^{\prime}}(\theta) \leqslant h_{F}(\theta), \quad \varphi<\theta<\psi .
$$

ДокАЗАТЕЛьСтво. Фиксируем $\theta \in(\varphi, \psi)$ и столь большое $r_{0}$, что при $r>r_{0}$ круг $(w:|w-z|<1)$, где $z=r e^{i \theta}$, попадает в сектор $\varphi<\arg z<\psi$. Пусть $\gamma-$ граница этого круга. Из формулы Коши следует, что

$$
\left|F^{\prime}(z)\right| \leqslant|F(w(z))|
$$

где $w(z)$ - некоторая точка $\gamma$. По свойству индикатора

$$
|F(w)|<\exp \left(|w|^{\rho}\left(h_{F}(\arg w)+\frac{\varepsilon}{2}\right)\right), \quad|w|>R_{0}(\varepsilon), \quad \varphi \leqslant \arg w \leqslant \psi
$$

Отсюда, из (21) и из непрерывности индикатора вытекает оценка

$$
\left|F^{\prime}(z)\right|<\exp \left((r+1)^{\rho}\left(h_{F}(\theta)+\varepsilon\right)\right), \quad r>r_{1},
$$

дающая требуемое неравенство (20). Лемма 11 доказана.

Встречающаяся далее константа $K(\beta, a)$ введена в начале $\S 3$.

Лемма 12 [7]. Пусть $p>0$. Тогда при $y \rightarrow+\infty$

$$
\int_{\mathbb{R}_{+}} \exp \left(p\left(y t-a t^{\alpha}\right)\right) d t \sim C y^{\beta / \alpha-\beta / 2} \cdot \exp \left(p K(\beta, a) y^{\beta}\right), \quad C=C(p, \alpha)>0 .
$$

Положим $e_{n}=e_{n}(t)\left(\right.$ см. (1)), $y_{n}=\operatorname{Im} \lambda_{n}, \varphi_{n}=\arg \lambda_{n}$. Норму в $B$ и $B_{+}$ обозначаем соответственно через $\|\cdot\|$ и $\|\cdot\|_{+}$. В лемме 13 для единообразия формулировок считаем, что показатель $p=\infty$ отвечает пространствам $B=C_{0}$ и $B_{+}=C_{0}^{+}$. Положительные константы $C$ в лемме 13 зависят от $B\left(B_{+}\right)$. 
Лемма 13. 1) $\left\|e_{n}\right\|,\left\|e_{n}\right\|_{+} \geqslant C\left(1+\left|y_{n}\right|\right)^{-1 / p}$,

2) $\left\|e_{n}\right\|_{+} \sim C y_{n}^{(\beta / \alpha-\beta / 2) / p} \exp \left(\left|\lambda_{n}\right|^{\beta} H\left(\varphi_{n}\right)\right)$, если $y_{n} \rightarrow+\infty$,

3) $\left\|e_{n}\right\| \sim C\left|y_{n}\right|^{(\beta / \alpha-\beta / 2) / p} \exp \left(\left|\lambda_{n}\right|^{\beta} H\left(\varphi_{n}\right)\right)$, если $y_{n} \rightarrow \pm \infty$.

ДокАзАтЕльство. Так как 3 ) следует из 1$), 2$ ), то надо доказать 1$), 2$ ). Пусть сначала $1 \leqslant p<\infty$. Так как $\left|e_{n}(t)\right|=\exp \left(y_{n} t-a|t|^{\alpha}\right)$, то

$$
\left\|e_{n}\right\|^{p},\left\|e_{n}\right\|_{+}^{p}>C \int_{0}^{1} \exp \left(p y_{n} t\right) d t=C \frac{\exp \left(p y_{n}\right)-1}{p y_{n}}
$$

и утверждение 1) верно. Утверждение 2) содержится в лемме 12.

Если $p=\infty$, т.е. $B=C_{0}, B_{+}=C_{0}^{+}$, то $\left\|e_{n}\right\|,\left\|e_{n}\right\|_{+} \geqslant 1$ и, значит, утверждение 1) верно. Утверждение 2) следует из свойства

$$
\max \left(\exp \left(y t-a t^{\alpha}\right): t \in \mathbb{R}_{+}\right)=\exp \left(K(\beta, a) y^{\beta}\right), \quad y>0,
$$

проверяемого дифференцированием. Лемма 13 доказана.

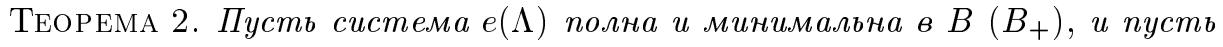
все точки $\lambda_{n}$, начиная с некоторой, просты. Тогда если система е $(\Lambda)$ равномерно минимальна в $B\left(B_{+}\right)$, то для индикатора $h_{F}(\theta)$ порождающей функиии $F(z)$ системы е $(\Lambda)$ в каждой точке $\theta_{0}$, предельной для последовательности $\varphi_{n}=\arg \lambda_{n}$, имеет место равенство

$$
h_{F}\left(\theta_{0}\right)=H\left(\theta_{0}\right) \quad\left(h_{F}\left(\theta_{0}\right)=H_{+}\left(\theta_{0}\right)\right)
$$

ДокАЗАТЕЛЬСтво. Предположим противное: $h_{F}\left(\theta_{0}\right)<H\left(\theta_{0}\right)\left(H_{+}\left(\theta_{0}\right)\right)$. Тогда, используя непрерывность индикатора и лемму 11 , заключаем, что при подходящем $\varepsilon>0$ и достаточно малом $\delta>0$ в случае пространства $B$ верна оценка

$$
h_{F^{\prime}}(\theta)<H(\theta)-2 \varepsilon, \quad\left|\theta-\theta_{0}\right| \leqslant \delta .
$$

Значит, при $\left|\theta-\theta_{0}\right| \leqslant \delta, r>r_{0}$

$$
\left|F^{\prime}\left(r e^{i \theta}\right)\right|<\exp \left(r^{\beta}\left(h_{F^{\prime}}(\theta)+\varepsilon\right)\right)<\exp \left(r^{\beta}(H(\theta)-\varepsilon)\right) .
$$

В частности, если $\left|\varphi_{n}-\theta_{0}\right| \leqslant \delta$, то

$$
\left|F^{\prime}\left(\lambda_{n}\right)\right|<\exp \left(\left|\lambda_{n}\right|^{\beta}\left(H\left(\varphi_{n}\right)-\varepsilon\right)\right) .
$$

Если речь идет о пространстве $B_{+}$, то в $(22) H\left(\varphi_{n}\right)$ заменяется на $H_{+}\left(\varphi_{n}\right)$. По определению точки $\theta_{0}$ неравенство (22) выполняется для некоторой последовательности индексов $n=n_{k} \rightarrow \infty$.

В дальнейшем нормы в пространствах $L^{q}, L_{+}^{q}, V, V_{+}$обозначаем соответственно через $\|\cdot\|_{q},\|\cdot\|_{q}^{+},\|\cdot\|_{V},\|\cdot\|_{V}^{+}$.

Пусть сначала $B=L^{p}$. Если $1<q \leqslant 2$, то из формул (19) по теореме Хаусдорфа-Юнга следует, что

$$
\left\|F_{n}(x)\right\|_{p} \leqslant C_{p}\left\|\exp \left(-a|t|^{\alpha}\right) h_{n}(t)\right\|_{q}
$$


и, значит,

$$
\left\|h_{n}\right\|_{q} \geqslant M_{p}\left\|F_{n}(x)\right\|_{p}
$$

Если $2<q \leqslant \infty$, то по неравенству Гёльдера

$$
\left\|\exp \left(-a|t|^{\alpha}\right) h_{n}(t)\right\|_{2} \leqslant C_{q}\left\|h_{n}(t)\right\|_{q}
$$

и, используя равенство Парсеваля, из (19) находим

$$
\left\|h_{n}\right\|_{q} \geqslant M_{p}\left\|F_{n}(x)\right\|_{2}, \quad 2<q \leqslant \infty .
$$

Пусть $B=C_{0}$. Тогда по замечанию 2 в $(19)$ вместо $h_{n}(t) d t$ присутствует $d h_{n}(t)$, где $h_{n}(t) \in V$. Очевидно,

$$
\left\|h_{n}\right\|_{V} \geqslant \operatorname{var} h_{n} \geqslant\left\|F_{n}(x)\right\|_{C(\mathbb{R})} .
$$

Если речь идет о пространстве $B_{+}$, то в полученных оценках (23)-(25) нормы $\left\|h_{n}\right\|_{q}$ и $\left\|h_{n}\right\|_{V}$ заменяются нормами $\left\|h_{n}\right\|_{q}^{+}$и $\left\|h_{n}\right\|_{V}^{+}$.

Итак, во всех рассматриваемых случаях мы получили однотипные оценки (23)(25) для норм элементов биортогональной системы. Поэтому конец доказательства достаточно провести для одного из этих случаев. Пусть для определенности $B=L^{p}\left(B_{+}=L_{+}^{p}\right), 2 \leqslant p<\infty$; тог да верна оценка $(23)$ с указанной выше заменой при переходе от $B$ к $B_{+}$.

Если $1<x \leqslant\left|\lambda_{n}\right|$, то

$$
\left|\frac{x}{x-\lambda_{n}}\right| \geqslant \frac{1}{2\left|\lambda_{n}\right|}
$$

и, значит, при $n>n_{1}$

$$
\left\|\frac{F(x)}{x-\lambda_{n}}\right\|_{p}=\left\|\frac{F(x)}{x} \frac{x}{x-\lambda_{n}}\right\|_{p} \geqslant \frac{1}{2\left|\lambda_{n}\right|}\left\|\frac{F(x)}{x}\right\|_{L^{p}\left(1,\left|\lambda_{n}\right|\right)}>\frac{C}{\left|\lambda_{n}\right|} .
$$

Отсюда, из (22), (23) следует, что в случае пространства $B$

$$
\left\|h_{n}\right\|_{q} \geqslant \frac{C}{\left|\lambda_{n}\right|} \exp \left(\left|\lambda_{n}\right|^{\beta}\left(\varepsilon-H\left(\varphi_{n}\right)\right)\right), \quad n=n_{k} \rightarrow \infty,
$$

а в случае пространства $B_{+}$в $(26)\left\|h_{n}\right\|_{q}$ и $H\left(\varphi_{n}\right)$ заменяются соответственно на $\left\|h_{n}\right\|_{q}^{+}$и $H_{+}\left(\varphi_{n}\right)$.

Если $\theta_{0} \neq 0, \pm \pi$, то из (26) и из утверждений 1$\left.)-3\right)$ леммы 13 вытекает, что

$$
\left\|e_{n}\right\| \cdot\left\|h_{n}\right\|,\left\|e_{n}\right\|_{+} \cdot\left\|h_{n}\right\|_{+}>C\left|\lambda_{n}\right|^{-2} \exp \left(\varepsilon\left|\lambda_{n}\right|^{\beta}\right), \quad n=n_{k} \rightarrow \infty,
$$

и критерий (4) равномерной минимальности нарушен.

Пусть $\theta_{0}=0, \pm \pi$; тогда $H\left(\varphi_{n}\right), H_{+}\left(\varphi_{n}\right) \rightarrow 0, n=n_{k} \rightarrow \infty$. По утверждению 1$)$ леммы $13\left\|e_{n}\right\|,\left\|e_{n}\right\|_{+}>C\left(1+\left|y_{n}\right|\right)^{-1}$, что вместе с (26) дает оценку

$$
\left\|e_{n}\right\| \cdot\left\|h_{n}\right\|>\frac{C}{\left|\lambda_{n}\right|^{2}} \exp \left(\left|\lambda_{n}\right|^{\beta}\left(\varepsilon-H\left(\varphi_{n}\right)\right)\right)>C_{1} \exp \left(\frac{\varepsilon}{2}\left|\lambda_{n}\right|^{\beta}\right), \quad n=n_{k}>N
$$

и аналогичную оценку для $\left\|e_{n}\right\|_{+} \cdot\left\|h_{n}\right\|_{+}$(в этом случае в $(27)$ появится $H_{+}\left(\varphi_{n}\right)$ вместо $H\left(\varphi_{n}\right)$, но на правую часть это не повлияет). Снова получено противоречие с (4). Теорема 2 доказана. 
ТЕОРЕМА 3. Пусть выполнены условия теоремы 2, и пусть порождающая

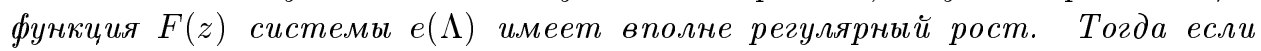
система е( $\Lambda)$ равномерно минимальна в $B\left(B_{+}\right)$, то

$$
h_{F}(\theta)=H(\theta) \quad\left(h_{F}(\theta)=H_{+}(\theta)\right), \quad-\pi \leqslant \theta \leqslant \pi,
$$

əде $h_{F}(\theta)-$ индикатор функиии $F(z)$.

ДоКАЗАТЕльСТво. Обозначив $h(\theta)=h_{F}(\theta)$, предположим противное. В силу теоремы 1 это будет означать, что в некоторой точке $\theta \in[-\pi, \pi]$ имеет место неравенство

$$
h(\theta)<H(\theta) \quad\left(h(\theta)<H_{+}(\theta)\right) .
$$

В силу непрерывности участвующих функций и по утверждению 1) теоремы 1 найдется интервал $(a, b)$ такой, что неравенство (29) вьполнено для всех его точек, а в точках $\theta=a, b$ имеет место равенство (28), причем $b-a \leqslant \pi / \beta$.

По теореме 2 ни одна точка интервала $(a, b)$ не является предельной для последовательности $\left(\arg \lambda_{n}\right)$. Отсюда в силу полной регулярности роста функции $F(z)$ следует $[17 ;$ гл. $3, \S 3]$, что на отрезке $[a, b]$ индикатор $h(\theta)$ есть $\beta$-тригонометрическая функция, т.е.

$$
h(\theta)=A \cos \beta(\theta-\gamma)
$$

где $A, \gamma$-некоторые числа. Убедимся, что равенство (28) при $\theta=a, b$ несовместимо с условием $b-a \leqslant \pi / \beta$.

Случай $A=0$ тривиален. Тогда $h(\theta)=H_{+}(\theta)$ на $[-\pi, 0]$, значит, в случае пространства $B_{+}$участвующая в $(29)$ точка $\theta \in(0, \pi)$. В случае пространства $B$ либо $\theta \in(0, \pi)$, либо $\theta \in(-\pi, 0)$. В обоих случаях $b-a=\pi>\pi / \beta$.

Пусть $A \neq 0$. В точках $\theta=0, \pm \pi$ равенство (28) невозможно. Действительно, если $h(\theta)=0$, то $h(t)>H(t)$ для некоторых точек $t$ из сколь угодно малой окрестности точки $\theta$, так как $H^{\prime}(\theta)=0$, тогда как $h^{\prime}(\theta) \neq 0$. Это дает противоречие с (12). По той же причине в случае пространства $B_{+}$равенство $(28)$ невозможно для точек $\theta \in[-\pi, 0]$. Значит, ввиду четности $H(\theta)$ достаточно рассмотреть случай равенства $(28)$ в точке $\theta^{*} \in(0, \pi)$.

Так как $h\left(\theta^{*}\right)>0$, то в силу (12) найдутся точки $\theta_{1}, \theta_{2} \in(0, \pi), \theta_{2}=\theta_{1}+\pi / \beta$, такие, что $h(\theta)>0$ на $\left(\theta_{1}, \theta_{2}\right)$ и $h\left(\theta_{1}\right)=h\left(\theta_{2}\right)=0$. Так как $H\left(\theta_{1}\right)>0$, то $h(\theta)<H(\theta)$ на $\left[\theta_{1}, \theta_{1}+\delta\right]$ при некотором $\delta>0$. На интервале $\left(\theta_{1}-\pi / \beta, \theta_{1}\right)$ это неравенство очевидно, так как на нем $h(\theta)<0$, а $H(\theta), H_{+}(\theta) \geqslant 0$. В итоге мы получили, что неравенство $h(\theta)<H(\theta)$ выполняется на интервале $\left(\theta_{1}-\pi / \beta, \theta_{1}+\delta\right)$ длины большей чем $\pi / \beta$. А это противоречит теореме 1 . Теорема 3 доказана.

\section{$\S 5$. Отсутствие базисов}

Рассмотрим систему

$$
\left(e^{-i \lambda_{n} t} \exp \left(-\frac{t^{2}}{2}\right)\right), \quad \Lambda=\left(\lambda_{n}\right)
$$


где

$$
\begin{gathered}
\Lambda=\bigcup_{k=0}^{3}\left(2 \sqrt{\pi(n+h)} \exp \left(i\left(\gamma+\frac{\pi}{2} k\right)\right)\right)_{n=1}^{\infty} \cup\left\{\lambda_{1}\right\} \cup\left\{\lambda_{2}\right\}, \\
h>-1, \quad \gamma \in\left[0,-\frac{\pi}{2}\right),
\end{gathered}
$$

причем точки $\lambda_{1} \neq \lambda_{2}$ не принадлежат предыдущему множеству в (31). Заметим, что система (30) отвечает случаю $\alpha=2$ в более общей системе (1). Первьй пример полной и минимальной системы (1) в $L^{2}$ с $\alpha=2$ Р. Залик и А.Т. Саад [7] нашли как раз в классе (30)-(31). А именно в [7] показано, что если $h=0, \gamma=\pi / 4$, то система (30)-(31) полна и минимальна в $L^{2}$. Этот результат был расширен Т. А. Сальниковой [9] и автором [18]; в итоге, верна следующая

Теорема Б. Система (30)-(31) полна и минимальна в $L^{p}, 2 \leqslant p<\infty$, тогда и только тогда, когда

$$
-\frac{1}{4 p}<h \leqslant \frac{1}{4 q}
$$

Доказательства в [7], [9], [18] сушественно использовали специфику показателя $\alpha=2$. В [10] для всех $\alpha>1, a>0$ был построен класс порождающих функций порядка $\beta$, зависяших от параметра $\mu \in \mathbb{C}$, и описаны значения $\mu$, при которых соответствующие системы $e(\Lambda)$ полны и минимальны в $L^{p}\left(L_{+}^{p}\right), 2 \leqslant p<\infty$. В статье [11] класс порождающих функций был расширен и результат [10] распространен на пространства $L^{p}, L_{+}^{p}, 1 \leqslant p<2$, и $C_{0}, C_{0}^{+}$. Сформулируем совокупный результат работ [10], [11] в той общей форме, в которой он приведен в [11]; отметим еще, что в [11] указаны опечатки, допущенные в [10].

Пусть $0<\left|\theta_{k}\right|<\pi, k=\overline{1, m}$. Положим $a_{k}=\left|\sin \theta_{k}\right|^{\beta-1}$ и

$$
\begin{aligned}
\theta_{k}^{0}=\frac{\theta_{k}}{\alpha}+\frac{\pi}{2 \beta}, & \text { если } \theta_{k} \in(0, \pi), \\
\theta_{k}^{0}=\frac{\theta_{k}}{\alpha}-\frac{\pi}{2 \beta}, & \text { если } \theta_{k} \in(-\pi, 0) .
\end{aligned}
$$

Введем функцию

$$
e(z)=\sum_{k=1}^{m} c_{k} E_{\beta}\left(a_{k}^{1 / \beta} z e^{-i \theta_{k}^{0}} ; \mu\right), \quad c_{k} \neq 0, \quad m \in \mathbb{N},
$$

где $E_{\rho}(z ; \mu)$ - функция типа Миттаг-Леффлера, и пусть

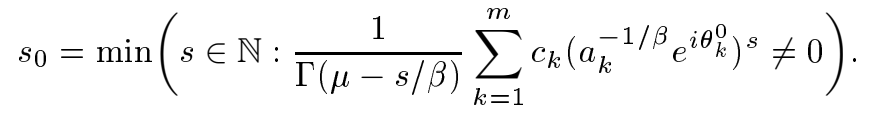

Рассматривается система $e(\Lambda)$ (см. (10)), для которой порождающей функцией является функция

$$
F(z)=e\left((K(\beta, a))^{1 / \beta} z\right)
$$

Для единообразия формулировок (и только в них) полагаем $L^{\infty}=C_{0}, L_{+}^{\infty}=C_{0}^{+}$. 
Теорема В. Пусть $-\pi<\theta_{1}<\cdots<\theta_{l}<0<\theta_{l+1}<\cdots<\theta_{m}<\pi$, причем

$$
\begin{gathered}
\theta_{1} \leqslant-\pi+\frac{\pi}{\beta}, \quad \theta_{m} \geqslant \pi-\frac{\pi}{\beta}, \quad \theta_{l} \geqslant-\frac{\pi}{\beta}, \quad \theta_{l+1} \leqslant \frac{\pi}{\beta}, \\
\theta_{k+1}-\theta_{k} \leqslant \frac{\pi}{\beta}, \quad k=1, \ldots, l-1, l+1, \ldots, m-1 .
\end{gathered}
$$

Пусть $s_{0}<\infty$, причем $s_{0} \geqslant 2$ при $p=1, \infty$. Тогда для полноты и минимальности системы $е(\Lambda)$ в $L^{p}, 1 \leqslant p \leqslant \infty$, необходимо и достаточно, чтобъ

$$
\begin{aligned}
\frac{1}{q \alpha}+\frac{1}{2} & <\operatorname{Re} \mu \leqslant \frac{1}{q \alpha}+\frac{1}{2}+\frac{1}{\beta} & \text { npu } & 1<p \leqslant \infty \quad u \\
\frac{1}{2} & \leqslant \operatorname{Re} \mu<\frac{1}{2}+\frac{1}{\beta} & \text { npu } & p=1 .
\end{aligned}
$$

ТеОрема Г. Пусть $0<\theta_{1}<\cdots<\theta_{m}<\pi$, причем

$$
\theta_{1} \leqslant \frac{\pi}{\beta}, \quad \theta_{m} \geqslant \pi-\frac{\pi}{\beta}, \quad \theta_{k+1}-\theta_{k} \leqslant \frac{\pi}{\beta}, \quad k=\overline{1, m-1} .
$$

Пусть $s_{0}<\infty$, причем $s_{0} \geqslant 2$ при $p=1, \infty$. Тогда для полноты и минимальности системы $е(\Lambda)$ в $L_{+}^{p}, 1 \leqslant p \leqslant \infty$, необходимо и достаточно выполнение условия (34).

Системами (30)-(31) и системами $e(\Lambda)$ с порождающей функцией $(33)$ к настоящему времени исчерпьвается класс известных полных и минимальных систем $e(\Lambda)$ в $L^{p}, L_{+}^{p}, 1 \leqslant p \leqslant \infty$. Выясним, выполняется ли для них необходимое условие равномерной минимальности, содержашееся в теореме 3.

Начнем с системы (30)-(31). Обозначим

$$
L(z)=z \prod_{n=1}^{\infty}\left(1-\frac{z^{2}}{(n+h)^{2}}\right)
$$

Хорошо известно (см., например, [17]), что $L(z)$ - целая функция экспоненциального типа и вполне регулярного роста с индикатором $h_{L}(\theta)=\pi|\sin \theta|$. Порождающая функция системы (30)-(31) имеет вид

$$
F(z)=\exp \left(-\frac{z^{2}}{4}\right) L\left(\left(\frac{z e^{-i \gamma}}{2 \sqrt{\pi}}\right)^{2}\right) \frac{\left(z-\lambda_{1}\right)\left(z-\lambda_{2}\right)}{z^{2}}
$$

(см. $[9],[16],[18])$. Из сказанного о функции $L(z)$ следует, что целая функция $F(z)$ порядка $\beta=2$ имеет вполне регулярный рост и ее индикатор есть

$$
h_{F}(\theta)=\frac{1}{4}|\sin 2(\theta-\gamma)|-\frac{1}{4} \cos 2 \theta
$$

Так как функция $H(\theta)$ всюду дифференцируема, а функция $h_{F}(\theta)$ не дифференцируема в точке $\theta=\gamma$, то равенство (28) невозможно. Значит, из теоремы 3 мы получаем 
СЛЕДСТВИЕ 1. В классе полных и минимальных в $L^{p}, 1 \leqslant p \leqslant \infty$, систем (30)-(31) нет равномерно минимальных систем.

Для рассмотрения систем $e(\Lambda)$ с порождающей функцией (33) воспользуемся леммой 9 . Из нее следует, что функция $E_{\beta}(z ; \mu)$ имеет порядок $\beta$ и вполне регулярньй рост, причем ее индикатор $h_{E}(\theta)$ есть $2 \pi$-периодическая функция такая, что $h_{E}(\theta)=0$ при $\pi /(2 \beta) \leqslant|\theta| \leqslant \pi$ и $h_{E}(\theta)=\cos \beta \theta$ при $|\theta| \leqslant \pi /(2 \beta)$.

В условиях теоремы В всегда $m \geqslant 2$. Если $1<\beta \leqslant 2$, то в теореме $Г$ набор $\theta_{k}$ может состоять из одной точки (например, $\left.\theta_{1}=\theta_{1}^{0}=\pi / 2\right)$. Тогда в $(32) m=1$, функции $e(z)$ и $E_{\beta}\left(a_{1}^{1 / \beta} z e^{-i \theta_{1}^{0}} ; \mu\right)$ пропорциональны и их индикаторы связаны соотношением $h_{e}(\theta)=a_{1} h_{E}\left(\theta-\theta_{1}^{0}\right)$. Из сказанного выше об индикаторе $h_{E}(\theta)$ следует, что индикатор $h_{e}(\theta)$, а значит, и индикатор $h_{F}(\theta)$ не является всюду дифференцируемой функцией. Значит, условие (28) не выполняется. Далее, при $\beta>1$ все корни функции $E_{\beta}(z ; \mu)$, а значит, и функции $F(z)$, за исключением, быть может, конечного числа, просты [19]. По теореме 3 в случае $m=1$ полные и минимальные системы $e(\Lambda)$ в $L_{+}^{p}$, фигурирующие в теореме $\Gamma$, не являются равномерно минимальньми.

Пусть $m \geqslant 2$. Рассмотрим $2 \pi$-периодическую функцию

$$
h_{k}(\theta)=a_{k} \cos \beta\left(\theta-\theta_{k}^{0}\right), \quad\left|\theta-\theta_{k}^{0}\right| \leqslant \frac{\pi}{2 \beta} ; \quad h_{k}(\theta)=0, \quad \frac{\pi}{2 \beta} \leqslant\left|\theta-\theta_{k}^{0}\right| \leqslant \pi .
$$

Функция $h_{k}(\theta)$ есть индикатор слагаемого с номером $k$ в сумме (32). Значит, из (32) и из определения числа $s_{0}$ следует, что функция $e(z)$ имеет порядок $\beta$ и вполне регулярный рост и ее индикатор

$$
h_{e}(\theta)=\max _{k=\overline{1, m}}\left(0, h_{k}(\theta)\right)
$$

Мы видим, что в условиях теорем В и Г индикатор $h_{e}(\theta)$, а значит, и индикатор $h_{F}(\theta)$ не является всюду дифференцируемой функцией. Следовательно, чтобы, сославшись на теорему 3 , с делать вывод об отсутствии равномерной минимальности у систем $e(\Lambda)$, фигурирующих в этих теоремах, остается доказать простоту всех корней функции $e(z)$, за исключением, быть может, конечного числа.

Пусть для определенности мы находимся в условиях теоремы $\Gamma$ (в случае теоремы В рассуждения аналогичны). По предположению $\theta_{k+1}-\theta_{k} \leqslant \pi / \beta$, a $\theta_{k}^{0}=\theta_{k} / \alpha+\pi /(2 \beta)$. Отсюда

$$
\theta_{k+1}^{0}-\theta_{k}^{0}=\frac{\theta_{k+1}-\theta_{k}}{\alpha}<\theta_{k+1}-\theta_{k} \leqslant \frac{\pi}{\beta}
$$

Значит, арки косинусоид - части графиков функций $h_{k}(\theta), h_{k+1}(\theta)$ с соседними номерами - пересекаются в некоторой точке с абсциссой $t_{k}$, причем $h_{k}\left(t_{k}\right)=$ $h_{k+1}\left(t_{k}\right)>0, k=\overline{1, m-1}$. Пусть $t_{0}, t_{m}$ - точки, в которых $h_{1}\left(t_{0}\right)=0, h_{m}\left(t_{m}\right)=0$ $\left(0<t_{0}<t_{m}<\pi\right)$.

Фиксируем достаточно малое $\varepsilon>0$. Утверждается, что вне секторов $U_{j}=$ $U_{j}(\varepsilon)=\left(\theta:\left|\theta-t_{j}\right|<\varepsilon\right), j=\overline{0, m}$, при $|z|>R$ нет корней $e(z)$. Сначала убедимся, что их нет при

$$
t_{m}+\varepsilon-2 \pi \leqslant \theta \leqslant t_{0}-\varepsilon, \quad|z|>R
$$


По лемме 9 в этом секторе

$$
e(z)=-\frac{A}{z^{s_{0}}}+O\left(\frac{1}{r^{s_{0}+1}}\right), \quad r \rightarrow \infty
$$

где

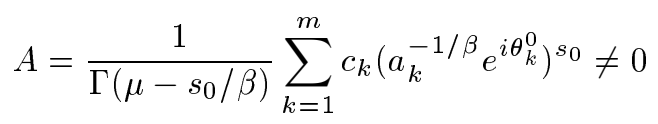

по смыслу числа $s_{0}$. Из (36) следует отсутствие у $e(z)$ корней на множестве $(35)$ при достаточно большом $R$.

Для сокрашения записи введем обозначения

$$
g(z)=\frac{1}{\beta} z^{\beta(\mu-1)} \cdot e(z), \quad C_{k}=c_{k} a_{k}^{1-\mu} e^{-i \beta(1-\mu) \theta_{k}^{0}}, \quad l=\beta(\mu-1)-s_{0} .
$$

По лемме 9

$$
g(z)=\sum_{k} C_{k} \exp \left(a_{k}\left(z e^{-i \theta_{k}^{0}}\right)^{\beta}\right)+\frac{A}{\beta} z^{l}+O\left(z^{l-1}\right), \quad r \rightarrow \infty
$$

где суммирование ведется по тем $k$, для которых $\left|\theta-\theta_{k}^{0}\right| \leqslant \gamma \in(\pi /(2 \beta), \pi / \beta)$. Множество индексов $k$ зависит от $\theta=\arg z$.

Пусть точка $z$ находится в секторе

$$
t_{j-1}+\varepsilon \leqslant \theta \leqslant t_{j}-\varepsilon, \quad j=\overline{1, m}, \quad|z|>R .
$$

Тогда в (37) обязательно присутствует индекс $k=j$. Далее, так как

$$
\left|\exp \left(a_{k}\left(z e^{-i \theta_{k}^{0}}\right)^{\beta}\right)\right|=\exp \left(r^{\beta} a_{k} \cos \beta\left(\theta-\theta_{k}^{0}\right)\right),
$$

а на множестве (38)

$$
a_{j} \cos \beta\left(\theta-\theta_{j}^{0}\right) \geqslant a_{k} \cos \beta\left(\theta-\theta_{k}^{0}\right)+\delta_{1}, \quad \delta_{1}>0, \quad k \neq j,
$$

то слагаемое с индексом $k=j$ доминирует в данном секторе, т.е.

$$
g(z)=C_{j} \exp \left(a_{j}\left(z e^{-i \theta_{j}^{0}}\right)^{\beta}\right)(1+o(1))+O\left(z^{l}\right), \quad r \rightarrow \infty .
$$

И так как в этом секторе

$$
\left|\exp \left(a_{j}\left(z e^{-i \theta_{j}^{0}}\right)^{\beta}\right)\right| \geqslant \exp \left(\delta r^{\beta}\right), \quad \delta>0,
$$

то из (39) следует отсутствие у функции $g(z)$, а значит, и у функции $e(z)$ корней в секторах (38). Мы доказали, что при $|z|>R$ все корни $e(z)$ лежат в секторах $U_{j}$, $j=\overline{0, m}$.

Пусть $j \neq 0, m$. В секторе $U_{j}(3 \varepsilon / 2)$ верна асимптотика $(37)$, причем если $\varepsilon$ достаточно мало, то множество индексов $k$ одно и то же для всех $z \in U_{j}(3 \varepsilon / 2)$. 
Известно [20; гл. 1, теорема 4.2], что асимптотика такого типа в каждом подсекторе допускает дифференцирование. Отсюда для $z \in U_{j}=U_{j}(\varepsilon)$

$$
g^{\prime}(z)=\beta z^{\beta-1} \sum_{k} C_{k} a_{k} e^{-i \beta \theta_{k}^{0}} \exp \left(a_{k}\left(z e^{-i \theta_{k}^{0}}\right)^{\beta}\right)+O\left(z^{l-1}\right), \quad r \rightarrow \infty .
$$

В ненулевых кратных корнях функции $e(z)$ левые части в $(37)$ и (41) обрашаются в нуль. Поэтому, деля (41) на $\beta z^{\beta-1} a_{j+1} e^{-i \beta \theta_{j+1}^{0}}$ и вычитая полученное из (37), видим, что в кратных корнях функции $e(z)\left(z \in U_{j}, z \neq 0\right)$ должно выполняться условие

$$
\begin{aligned}
& C_{j}\left(1-\frac{a_{j} e^{-i \beta \theta_{j}^{0}}}{a_{j+1} e^{-i \beta \theta_{j+1}^{0}}}\right) \exp \left(a_{j}\left(z e^{-i \theta_{j}^{0}}\right)^{\beta}\right) \\
& \quad+\sum_{k \neq j, j+1} B_{k} \exp \left(a_{k}\left(z e^{-i \theta_{k}^{0}}\right)^{\beta}\right)+O\left(z^{l-1}\right)=0,
\end{aligned}
$$

где $B_{k}$ - некоторые коэффициенты. Убедимся, что коэффициент $B_{j}$ при экспоненте в первом слагаемом отличен от нуля. Так как $C_{j} \neq 0$, то достаточно проверить, что

$$
a_{j+1} e^{i \beta \theta_{j}^{0}} \neq a_{j} e^{i \beta \theta_{j+1}^{0}} .
$$

Предположим противное: здесь имеет место знак равенства. Так как $a_{j}, a_{j+1}>0$, то отсюда $\beta \theta_{j+1}^{0}=\beta \theta_{j}^{0}+2 \pi n$ при некотором $n \in \mathbb{N}$. Тогда $\theta_{j+1}-\theta_{j}=\alpha\left(\theta_{j+1}^{0}-\theta_{j}^{0}\right)=2 \pi n \alpha / \beta>2 \pi / \beta$, а это противоречит тому, что $\theta_{j+1}-\theta_{j} \leqslant \pi / \beta$ по условию теоремы $Г$. Итак, $B_{j} \neq 0$.

Из тех же соображений, что применялись при рассмотрении сектора (38), следует, что если $\varepsilon$ достаточно мало, а $z \in U_{j}, j \neq 0, m$, то в (42) при $r \rightarrow \infty$ доминирует первое слагаемое, т.е. (42) записывается в виде

$$
B_{j}(1+o(1)) \exp \left(a_{j}\left(z e^{-i \theta_{j}^{0}}\right)^{\beta}\right)+O\left(z^{l-1}\right)=0, \quad r \rightarrow \infty .
$$

Но при достаточно малом $\varepsilon$ для $z \in U_{j}$ верна оценка (40). Значит, необходимое условие (43) кратного корня функции $е(z)$ не может выполняться для сколь угодно больших $|z|, z \in U_{j}, j \neq 0, m$.

Осталось рассмотреть секторы $U_{j}, j=0, m$. Если $\varepsilon$ достаточно мало, то в секторе $U_{0}$ верна асимптотика (37) с единственным индексом $k=1$. Значит, при $|z|>R$ корни $e(z)$ в секторе $U_{0}$ совпадают с корнями функции

$$
C z^{-l} \exp \left(a_{1}\left(z e^{-i \theta_{1}^{0}}\right)^{\beta}\right)-A+O\left(\frac{1}{r}\right)
$$

где $C$ - соответствующая константа. Но именно такая функция встречается в [19] при исследовании асимптотики корней функции Миттаг-Леффлера, где доказывается [19; с. 122], что все достаточно большие по модулю корни функции (44)просты. Итак, при $|z|>R$ все корни функции $e(z)$ в секторе $U_{0}$ просты. Аналогично это утверждение доказывается для сектора $U_{m}$.

Мы доказали простоту всех достаточно больших по модулю корней порождаюшей функции (33), и из теоремы 3 вытекает 
СлЕДСТВИЕ 2. Полные и минимальные в $L^{p}\left(L_{+}^{p}\right), 1 \leqslant p \leqslant \infty$, cuстемы е $(\Lambda)$, описываемые теоремой $\mathrm{B}$ (теоремой $Г$ ), не являются равномерно минимальньмми.

Следствия 1, 2 показывают, что среди имеюшихся полных и минимальных систем $e(\Lambda)$ в пространствах $L^{p}, L_{+}^{p}, 1 \leqslant p<\infty, C_{0}, C_{0}^{+}$равномерно минимальных систем нет. Подавно среди них нет и базисов.

\section{Список литературы}

1. Винер H. Интеграл Фурье и некоторые его приложения. М.: Физматгиз, 1963.

2. Седлецкий A. M. Аппроксимация сдвигами функции на прямой // Теория приближения функций. Труды Междунар. конф. приближ. функций. Киев, 31 мая - 5 июня 1983 г. М., 1987. C. $397-400$.

3. Zalik R. On approximation by shifts and a theorem of Wiener // Trans. Amer. Math. Soc. 1978. V. 243. P. 299-308.

4. Zalik R. On some gap theorems and the closure of translates // Notices Amer. Math. Soc. 1978. V. 25. № 2. P. A-314.

5. Faxen B. On approximation by translates and related problems in function theory // Ark. Mat. 1981. V. 19. № 2. P. 271-289.

6. Седлецкий A.M. Аппроксимация сдвигами и полнота взвешенных систем экспонент в $L^{2}(\mathbb{R}) / /$ Матем. сб. 1984. Т. 123. № 1. С. 92-107.

7. Zalik R, Saad A.T. Some theorems concerning holomorphic Fourier transforms // J. Math. Anal. Appl. 1987. V. 126. P. 483-493.

8. Сальникова T. А. Полные и минимальные системы экспонент в пространствах $L^{2}(\mathbb{R}) / /$ Матем. заметки. 1994. Т. 55. №3. С. 118-129.

9. Сальникова T. A. Полные и минимальные системы экспонент в лебеговых пространствах на вещественной оси // Дисс. ... канд. физ.-матем. наук. М.: РУДН, 1995.

10. Седлецкий A. M. Аппроксимативные свойства систем экспонент на прямой и полупрямой // Матем. сб. 1998. Т. 89. № 3. С. 125-140.

11. Седлецкий A. M. Аппроксимация посредством экспонент на прямой и на полупрямой // Anal. Math. 2001. T. 27.

12. Крейн С. Г. (ред.) Функциональньй анализ. М.: Наука, 1972.

13. Седлецкий A. М. Аппроксимативные свойства систем экспонент в $L^{p}(a, b) / /$ Дифференц. уравнения. 1995. Т. 31. № 5. С. 1675-1681.

14. Федорюк М. В. Метод перевала. М.: Наука, 1977.

15. Джсрбашян M. М. Интегральные преобразования и представления функций в комплексной области. М.: Наука, 1966.

16. Седлецкий A. М. Преобразования Фурье быстро убьвающих функций // Изв. РАН. Сер. матем. 1997. Т. 61. №3. С. 187-202.

17. Левин Б. Я. Распределение корней целых функций. М.: ГИТТЛ, 1956.

18. Sedletskii A. M. Fourier transforms and approximations. Amsterdam: Gordon and Breach, 2000.

19. Седлецкий A. М. Асимптотические формулы для нулей функции типа Миттаг- Леффиера // Anal. Math. 1994. T. 20. C. 117-132.

20. Олвер $\Phi$. Введение в асимптотические методы и специальные функции. М.: Наука, 1978.

Московский государственный

университет им. М.В. Ломоносова
Поступила в редакцию

04.04.2001 